\title{
The emergence of coherence in complex networks of heterogeneous dynamical systems
}

\author{
Juan G. Restrepo, ${ }^{1,2, \text { : Edward Ott, },^{1,3} \text { and Brian R. Hunt }}{ }^{2,4}$ \\ 1 Institute for Research in Electronics and Applied Physics, \\ University of Maryland, College Park, Maryland 20742, USA \\ 2 Department of Mathematics, University of Maryland, College Park, Maryland 20742, USA \\ ${ }^{3}$ Department of Physics and Department of Electrical and Computer Engineering, \\ University of Maryland, College Park, Maryland 20742, USA \\ 4 Institute for Physical Science and Technology, University of Maryland, College Park, Maryland 20742, USA
}

(Dated: June 20, 2018)

\begin{abstract}
We present a general theory for the onset of coherence in collections of heterogeneous maps interacting via a complex connection network. Our method allows the dynamics of the individual uncoupled systems to be either chaotic or periodic, and applies generally to networks for which the number of connections per node is large. We find that the critical coupling strength at which a transition to synchrony takes place depends separately on the dynamics of the individual uncoupled systems and on the largest eigenvalue of the adjacency matrix of the coupling network. Our theory directly generalizes the Kuramoto model of equal strength, all-to-all coupled phase oscillators to the case of oscillators with more realistic dynamics coupled via a large heterogeneous network.
\end{abstract}

PACS numbers: 05.45.-a, 05.45.Xt, 89.75.-k

In recent years, much progress has been made in describing the complex structure of real world networks [1, 2]. The study of dynamical processes taking place in such complex networks has applications in fields ranging from biology to engineering. One of the most important phenomena involving networks of coupled dynamical systems is the emergence of large-scale coherent behavior [3, 4]. It is often observed that large collections of heterogeneous dynamical systems (e.g., cells, fireflies) synchronize their rhythms so that a significant proportion of the systems have states that are highly correlated with those of the others. It is natural to ask what determines the emergence of such coordinated behavior given the network of interactions between the dynamical systems and their individual dynamics.

The case of equal-strength, all-to-all coupled phase oscillators was studied by Kuramoto [5], who considered the case of $N$ oscillators, each of which is described by a phase $\theta_{j}$ and an intrinsic frequency $\omega_{j}$. Kuramoto assumed sinusoidal coupling so that the phase of oscillator $j$ evolves as $\dot{\theta}_{j}=\omega_{j}+k \sum_{m=1}^{N} \sin \left(\theta_{m}-\theta_{j}\right)$. Kuramoto found that, in the limit $N \rightarrow \infty$, for coupling strengths $k$ less than a critical coupling strength $k_{c}$ that depends on the distribution of frequencies, the phases of the oscillators are incoherent, i.e., $\theta_{j}$ are uniformly distributed on $[0,2 \pi)$. For values of the coupling strength $k$ larger than $k_{c}$, a significant fraction of the oscillators evolve with a common frequency. The Kuramoto model has become a classical paradigm for the emergence of coherent behavior in an ensemble of heterogeneous oscillators (see [6] for reviews).

Although the Kuramoto model has the advantage that it can be treated analytically, it depends on the sys-

*Electronic address: juanga@math.umd.edu tem being simple in two major aspects. First, the network is assumed to be all-to-all, so that every oscillator is coupled with uniform strength to every other oscillator. Second, the dynamics and coupling term are highly simplified: each oscillator is described only by its phase and the coupling term is sinusoidal. Recently, collections of coupled dynamical systems which have either a more general interaction network or a more general dynamics have been studied. In previous works [7, 8] (see also 9, 10, 11, 12, 13]), we have studied the Kuramoto phase oscillator model generalized to the case of a general interaction network described by an adjacency matrix. We found that there is still a transition to synchrony at a critical coupling strength that depends on the largest eigenvalue of the adjacency matrix and the distribution of frequencies. On the other hand, 'globally' coupled (i.e., equal coupling strength, all-to-all) collections of many dynamical systems with more general dynamics (e.g., mixed collections of chaotic and periodic oscillators, chaotic maps, etc) have recently been studied [14, 15, 16, 17, 18], and a transition to synchrony has been observed at coupling strengths that depend on the dynamics of the uncoupled systems.

Our aim in this Letter is to generalize these previous works by studying large collections of heterogeneous general dynamical systems coupled by networks with complex topology. We find that in this general case there is a transition to coherence, and that the coupling strengths at which it occurs can be obtained from the uncoupled individual unit dynamics and the eigenvalues of the adjacency matrix. Thus, we achieve a separation of the problem into a part depending on the network only and a part depending on the individual unit dynamics only [19]. Our model allows strong heterogeneity and different dynamics in the collection of dynamical systems, making it potentially appropriate to describe biological or other strongly heterogeneous systems. 
We study networks of $N$ coupled maps satisfying

$$
\begin{gathered}
x_{n+1}^{(j)}=f\left(x_{n}^{(j)}, \mu_{j}\right)+w_{n}^{(j)}+ \\
k g\left(x_{n}^{(j)}\right) \sum_{m=1}^{N} A_{j m}\left[q\left(x_{n}^{(m)}\right)-\langle q(x)\rangle\right] .
\end{gathered}
$$

Here $j=1,2, \ldots, N$ labels the map and we are interested in large $N, f\left(x_{n}^{(j)}, \mu_{j}\right)$ determines the uncoupled dynamics of each individual map with a parameter $\mu_{j}$; for each map $j$ the vector of parameters $\mu_{j}$ is chosen randomly and independently of $j$ with a probability distribution $p(\mu)$; and the term $w_{n}^{(j)}$ is a random noise which is assumed to be statistically independent of $j$ and $n$ and to satisfy $E\left[w_{n}^{(j)}\right]=0, E\left[w_{n}^{(j)} w_{m}^{(l)}\right]=\sigma^{2} \delta_{n m} \delta_{j l}$, where $E[\cdot]$ represents the expected value. In the coupling term, $k$ is a global coupling strength and the scalar functions $g$ and $q$ are assumed to be smooth and bounded. The notation $\langle\cdot\rangle$ represents the average over the distribution of the vector of parameters $\mu_{i}$ and over the natural measures of the attractors of the noisy uncoupled $(k=0)$ system. Alternatively, $\langle q\rangle$ is the $\mu$-average of the infinite time average of $q\left(x_{n}\right)$ over a typical orbit $x_{n}$ of the noisy uncoupled system. We remark that $\langle q\rangle$ is independent of time. We also note that $x$ can be a vector for the situation of multidimensional individual maps. For simplicity, in what follows we consider $x$ to be a scalar.

The $N \times N$ matrix $A_{j m}$ determines the network of interactions: node $m$ interacts with node $j$ only if $A_{j m} \neq$ 0 . We refer to those nodes $m$ for which $A_{j m} \neq 0$ as the neighbors of node $j$, and to $d_{j}=\sum_{m=1}^{N} A_{j m}$ as the degree of node $j$.

We are interested in studying system (1) for the case in which nodes have a large number of neighbors. In this case, if the initial conditions are chosen distributed according to the natural measure of the attractors of the uncoupled systems, then, because of the large number of terms in the sum in the coupling term in Eq. (10), the fact that $x_{n}$ are distributed according to the measure of the uncoupled attractors, and the lack of correlations between the parameter vectors and the network, we can approximate

$$
\sum_{m=1}^{N} A_{j m} q\left(x_{n}^{(m)}\right) \approx\langle q(x)\rangle \sum_{m=1}^{N} A_{j m},
$$

and equality of these sums applies in the limit of an infinite number of neighbors. We refer to this situation as the incoherent state, and we will study in what follows its linear stability. Under the previously mentioned assumption of a large number of neighbors per node, the incoherent state is (approximately) a solution of the system (11). Its linear stability can be studied using the same methods that were used in Ref. 17] for the all-toall case. In the following, we will adapt these techniques to the case of general connectivity.
In order to study the linear stability of the incoherent state, we assume that $x_{n}^{(i)}$ is in the incoherent state and introduce an infinitesimal perturbation $\delta x_{n}^{(i)}$. Linearization of Eq. (11) produces

$$
\delta x_{n+1}^{(i)}=f^{\prime}\left(x_{n}^{(i)}, \mu_{i}\right) \delta x_{n}^{(i)}+k g\left(x_{n}^{(i)}\right) \sum_{j=1}^{N} A_{i j} q^{\prime}\left(x_{n}^{(j)}\right) \delta x_{n}^{(j)} .
$$

In order to solve Eq. (3) we consider (as in the variation of parameters method for differential equations) a perturbation $\epsilon_{n}^{(i)}$ of the uncoupled system $\epsilon_{n+1}^{(i)}=f^{\prime}\left(x_{n}^{(i)}, \mu_{i}\right) \epsilon_{n}^{(i)}$ with $\epsilon_{0}=1$. Defining $\Gamma_{n}^{(i)}=\sum_{j=1}^{N} A_{i j} q^{\prime}\left(x_{n}^{(j)}\right) \delta x_{n}^{(i)}$ and assuming exponential growth, so that $\Gamma_{n}^{(i)}=\gamma^{(i)} \eta^{n}$, we obtain for large $n$ [20]

$$
\gamma^{(m)}=k \sum_{i=1}^{N} A_{m i} \gamma^{(i)} \sum_{p=0}^{n} \frac{q^{\prime}\left(x_{n+1}^{(i)}\right) \epsilon_{n+1}^{(i)} g\left(x_{p}^{(i)}\right) \eta^{p-n-1}}{\epsilon_{p+1}^{(i)}} .
$$

In order to proceed further, we will again use the assumptions of large number of neighbors per node and statistical independence of the network and the vector of parameters. As we did in Eq. (2), we approximate Eq. (4) by

$\gamma^{(m)}=k\left\langle\sum_{p=0}^{n} \frac{q^{\prime}\left(x_{n+1}^{(i)}\right) \epsilon_{n+1}^{(i)} g\left(x_{p}^{(i)}\right) \eta^{p-n-1}}{\epsilon_{p+1}^{(i)}}\right\rangle \sum_{i=1}^{N} A_{m i} \gamma^{(i)}$.

[If the sum over $i$ in Eq. (4) is imagined as approximating $N$ times the expected value of a product of two random variables, Eq. (5) approximates $N$ times the product of the two expected values as suggested by our assumption of their independence.]

Let $Q(\eta)$ be the average in Eq. (5). With $m=n-p$, and letting $n \rightarrow \infty$,

$$
Q(\eta)=\eta^{-1}\left\langle\sum_{m=0}^{\infty} \frac{\epsilon_{n+1}}{\epsilon_{n-m+1}} q^{\prime}\left(x_{n+1}\right) g\left(x_{n-m}\right) \eta^{-m}\right\rangle .
$$

This quantity depends only on the dynamics of the individual uncoupled oscillators, and also results from the analysis of the globally coupled case [17]. From Eq. (51) we obtain $u^{(j)}=k_{c} \lambda_{j} Q(\eta) u^{(j)}$, where $u^{(j)}$ and $\lambda_{j}$ denote, respectively, the eigenvectors of $A$ and their corresponding eigenvalues. The onset of instability of the incoherent state corresponds to $|\eta|=1$, or $\eta=e^{i \omega}$. Thus, network mode $j$ becomes unstable at a critical coupling strength satisfying

$$
1=k_{c} \lambda_{j} Q\left(e^{i \omega}\right),
$$

where the critical frequency $\omega$ is found by $\operatorname{Im}\left[\lambda_{j} Q\left(e^{i \omega}\right)\right]=0$, and $\operatorname{Im}$ denotes the imaginary part. We are interested in the solutions $k_{c}$ of Eq. (7) with the smallest magnitude. Typically, they correspond to the mode associated to the eigenvalue of largest magnitude, which is usually real 21]. In this case the critical frequency is found from $\operatorname{Im}\left[Q\left(e^{i \omega}\right)\right]=0$ and is independent of the network. 
We remark that the definition and numerical computation of $Q(\eta)$ is in general nontrivial, since for $|\eta|=1$ (necessary for investigation of the onset of coherence) the individual terms in the sum in Eq. (6) diverge with $m$ for typical initial condition in chaotic maps. However, for large enough $|\eta|$ these terms decay exponentially with increasing $m$ and consequently the sum and average can be interchanged, so that

$$
Q(\eta)=\eta^{-1} \sum_{m=0}^{\infty}\left\langle\frac{\epsilon_{n+1}}{\epsilon_{n-m+1}} q^{\prime}\left(x_{n+1}\right) g\left(x_{n-m}\right)\right\rangle \eta^{-m} .
$$

In Refs. 17, 18] it is argued that the averages in the summand of (8) decay exponentially with $m$, so that (8) can be analytically continued to $|\eta|=1$ as desired.

We note that the function $Q(\eta)$ depends exclusively on the dynamics of the uncoupled oscillators and their parameter distribution function $p(\mu)$, while the eigenvalues $\lambda_{j}$ depend exclusively on the network. Therefore, an independent treatment of these problems allows determination of the critical coupling strengths for the full system given by Eq. (1).

We now present two examples illustrating our theory. In order to quantify the coherence, we define an order parameter $r$ by

$$
r^{2}=\left\langle\left(\frac{\sum_{m=1}^{N} \bar{d}_{m}\left[q\left(x_{n}^{(m)}\right)-\langle q(x)\rangle\right]}{\sum_{m=1}^{N} \bar{d}_{m}}\right)^{2}\right\rangle_{t}
$$

where $\langle\cdot\rangle_{t}$ denotes a time average and the in-degree $\bar{d}_{j}$ is defined by $\bar{d}_{m}=\sum_{j=1}^{N} A_{j m}$. Note that the numerator can be written as $\sum_{j=1}^{N} \sum_{m=1}^{N} A_{j m}\left[q\left(x_{n}^{(m)}\right)-\langle q(x)\rangle\right]$, and, therefore, $r$ measures the $r m s$ of the coupling term in Eq. (1) [aside from the factor $g\left(x^{(j)}\right)$ ]. Thus, the incoherent state corresponds to $r \approx 0$. We will investigate what happens to $r$ as the coupling strength $k$ is increased past the critical values predicted by the theory.

In our numerical experiments, we compute the order parameter $r$ using Eq. (9) with $x_{n}^{(m)}$ obtained from iteration of Eq. (11). We calculate the time average using 1000 iterations after the initial transients have disappeared. As an example, we consider [17] for the functions in Eq. (1), $f\left(x_{n}^{(i)}, \mu_{i}\right)=2 x_{n}^{(i)}+\mu_{i}, q(x)=\cos x$, and $g(x)=\sin (2 x)+\sin (4 x)$. Throughout $x$ is regarded as an angle-like variable and its value modulo $2 \pi$ to be taken where appropriate. In Ref. [17] $Q\left(e^{i \omega}\right)$ was calculated for Gaussian noise with mean zero and standard deviation $\sigma$ to be

$$
Q\left(e^{i \omega}\right)=-\frac{1}{2}\left(\frac{e^{-\sigma^{2} / 2}}{e^{i \omega}}\langle\cos (\mu)\rangle+2 \frac{e^{-5 \sigma^{2} / 2}}{e^{2 i \omega}}\langle\cos (3 \mu)\rangle\right),
$$

We numerically consider the following two examples, listed below with the theoretical critical coupling strengths obtained using expression (10) in Eq. (7).

1. Identical noisy maps with $\sigma=0.4, p(\mu)=\delta(\mu)$. For this example the solutions of Eq. (7) are $k_{c} \lambda_{j} \approx$ 1.49 and $k_{c} \lambda_{j} \approx-0.88$.

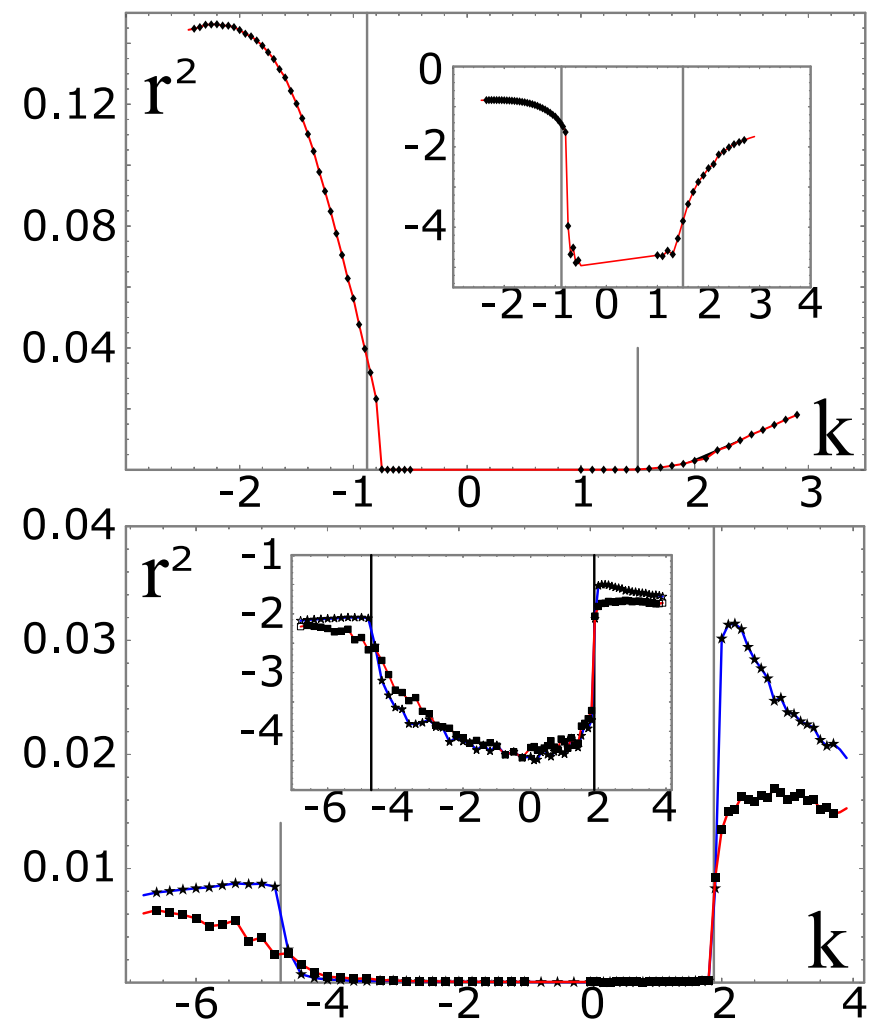

FIG. 1: The order parameter, $r^{2}$, plotted on a linear scale as a function of the coupling strength $k$. The insets show the same data plotted on a logarithmic scale for $r^{2}$. (a) Example 1 (identical noisy maps) with a scale-free network with $N=10^{5}$, exponent -3 and $d_{\min }=100$. (b) Example 2 (heterogeneous noiseless maps) with a scale-free network with $N=20000$, exponent -2.5 , and and lower cutoff $d_{\min }=50$ (boxes) and $d_{\min }=200$ (stars). The vertical lines indicate the theoretical values for the critical coupling strength.

2. Heterogeneous noiseless maps with $\sigma=0, p(\mu)=$ $2 / \pi$ if $0 \leq \mu<\pi / 2,0$ otherwise. In this example we obtain $k_{c} \lambda_{j}=3 \pi / 5$ and $k_{c} \lambda_{j}=-3 \pi / 2$.

For the network connectivity, we consider a scale free network with exponent $\gamma$, i.e., a network in which the degree distribution $P(d)$ satisfies $P(d) \propto d^{-\gamma}$ for $d \geq$ $d_{\text {min }}$ and 0 otherwise. We impose a lower cutoff $d \geq d_{\text {min }}$ so that our assumption of a large number of neighbors per node is satisfied. We use $\gamma=3, d_{\min }=100$ for example 1 and $\gamma=2.5, d_{\text {min }}=50$ and $d_{\text {min }}=200$ for example 2 . In order to construct such networks we use the Random Graph model of Chung et al. [22].

For scale free networks as described above, the largest positive eigenvalue $\lambda_{+}$is significantly larger than the magnitude of the most negative eigenvalue $\lambda_{-}$, and so the mode desynchronizing first is the one associated to the largest positive eigenvalue. Consequently, the value of $\lambda_{j}$ used to determine the critical coupling strengths $k_{c}$ for these examples will be $\lambda_{+}$.

In Figs. 1(a) and (b) we show the square of the order parameter as a function of $k$ for examples 1 and 2 with $N=10^{5}$ and $N=20000$, respectively (the insets show 
the same quantity on a logarithmic scale). For example 1 [Fig. 11(a)] the order parameter grows for values of the coupling strength close to the positive and negative critical values predicted by the theory (vertical lines in the figures). For example 2 [Fig. 1(b)] the transition on the positive side is quite sharp and occurs very close to the theoretical value $k_{c 1} \approx 1.88$, while on the negative side the transition, although somewhat less well defined, also occurs close to the theoretical critical coupling strength $k_{c 2} \approx-4.71$. On the negative side, the transition is not so sharp. However, we observe that as we increase $d_{\text {min }}$ from 50 (boxes) to 200 (stars), the transition becomes sharper. Generally, we find that the agreement with the theoretical results improves as the minimum degree $d_{\text {min }}$ and $N$ become larger [23]. The plots in Fig. 1 were produced by starting in the incoherent state and increasing the magnitude of the coupling strength $k$ from zero. We have found that the transitions in our examples are not hysteretic, i.e., the same behavior is observed if we use the synchronized state as an initial condition and decrease the magnitude of the coupling strength. We note that in the globally coupled case, it was found that the computation of $Q(\eta)$ fails to converge for an ensemble of identical noiseless logistic maps [17]. It was argued that this results from structural instability of the map and singularities in its invariant density, which make a perturbation approach questionable. Since the definition and numerical determination of $Q(\eta)$ in our case and for the globally coupled case are identical, the lack of convergence observed for this example in the globally coupled case will also occur in the case of a network. However, we note that a small amount of either noise or parameter heterogeneity was shown in [17] to restore the validity of the results.

In summary, we have studied the onset of synchronization in large networks of coupled maps (the case of coupled continuous time oscillators can also be treated by these methods and will be discussed elsewhere). We have found that the critical coupling strength at which the transition to synchrony takes place depends separately on the dynamics of the individual uncoupled oscillators and on the largest eigenvalue of the adjacency matrix of the network. Thus, we have achieved a separation of the problem of the stability of the incoherent state for networks of coupled dynamical systems into a part depending on the dynamics of the uncoupled individual units and a part depending exclusively on the network [19]. Our theory directly generalizes the Kuramoto model of equal strength, all-to-all coupled phase oscillators to the case of oscillators with more realistic dynamics coupled in a potentially complex network. The results we obtain suggest that knowledge of network properties that favor larger maximum eigenvalues can be used to promote synchronism in large networks of coupled dynamical systems.

This work was sponsored by ONR (Physics) and by NSF (DMS 0104087 and PHY 0456240).
[1] M. E. J. Newman, SIAM Review 45, 167 (2003).

[2] A. -L. Barabási, and R. Albert, Rev. Mod. Phys. 74, 47 (2002).

[3] A. Pikovsky, M.G. Rosenblum, and J. Kurths, Synchronization: A universal concept in nonlinear sciences, (Cambridge University Press, Cambridge, 2001).

[4] E. Mosekilde, Y. Maistrenko, and D. Postnov, Chaotic Synchronization: Applications to Living Systems (World Scientific, Singapore, 2002).

[5] Y. Kuramoto, Chemical Oscillations, Waves, and Turbulence, (Springer-Verlag, Berlin, 1984).

[6] S. H. Strogatz, Physica D 143, 1 (2000); E. Ott, Chaos in Dynamical Systems, Second edition, Sec. 6.5 (Cambridge University Press, New York, 2002).

[7] J. G. Restrepo, E. Ott, and B. R. Hunt, Phys. Rev. E 71, 036151 (2005).

[8] J. G. Restrepo, E. Ott, and B. R. Hunt, Chaos 16, 015107 (2006).

[9] T. Ichinomiya, Phys. Rev. E 70, 026116 (2004).

[10] D.-S. Lee, Phys. Rev. E 72, 026208 (2005).

[11] T. Ichinomiya, Phys. Rev. E 72, 016109 (2005).

[12] A. Jadbabaie, N. Motee, and M. Barahona. Proceedings of the American Control Conference (ACC 2004).

[13] Y. Moreno and A. E. Pacheco, Europhys. Lett. 68, 603 (2004).

[14] A. Pikovsky, M.G. Rosenblum, and J. Kurths, Europhys. Lett. 34, 165 (1996).

[15] H. Sakaguchi, Phys. Rev. E 61, 7212 (2000).

[16] D. Topaj, W.-H. Kye, and A. Pikovsky, Phys. Rev. Lett. 87, 074101 (2001).
[17] S.-J. Baek and E. Ott, Phys. Rev. E 69, 066210 (2004).

[18] E. Ott, P. So, E. Barreto, and T. Antonsen, Physica D 173, 29, (2002).

[19] In this respect our result is in the same spirit as that of the 'master stability function' of L.M. Pecora and T.L. Carroll [Phys. Rev. Lett. 80, 2109 (1998)]. The main difference is that we consider the onset of synchronism from an incoherent state of many potentially different chaotic or periodic systems, while Pecora and Carroll consider the stability of a fully synchronized state of identical oscillators.

[20] The derivation follows closely the all-to-all case in Ref. [17], which assumes $A_{n m}=1$. Further details will be published elsewhere.

[21] For symmetric matrices or matrices with positive entries the eigenvalue with largest magnitude is real. For a large class of nonsymmetric matrices with mixed positive/negative entries, we have found that if the average of the nonzero elements of the matrix is large enough, the eigenvalue with largest magnitude is also real and it is well separated from the eigenvalue with next largest magnitude. For details, see the Appendix of Ref. [8].

[22] F. Chung, L. Lu and V. Vu, Proc. Natl. Acad. Sci., 100, 6313 (2003).

[23] A quantitative treatment and theory for the effect of $d_{\text {min }}$ is given in 7] for the case of the Kuramoto model, and we expect similar behavior when the dynamics of $f$ at different parameter values are sufficiently similar. 